\title{
Fracture Behavior of Multidirectional DCB Specimen: Higher-Order Beam Theories
}

\author{
B. K. Raghu Prasad ${ }^{1}$ and D. V. T. G. Pavan Kumar ${ }^{2}$
}

\begin{abstract}
Mathematical models, for the stress analysis of symmetric multidirectional double cantilever beam (DCB) specimen using classical beam theory, first and higher-order shear deformation beam theories, have been developed to determine the Mode I strain energy release rate (SERR) for symmetric multidirectional composites. The SERR has been calculated using the compliance approach. In the present study, both variationally and nonvariationally derived matching conditions have been applied at the crack tip of DCB specimen. For the unidirectional and cross-ply composite DCB specimens, beam models under both plane stress and plane strain conditions in the width direction are applicable with good performance where as for the multidirectional composite DCB specimen, only the beam model under plane strain condition in the width direction appears to be applicable with moderate performance. Among the shear deformation beam theories considered, the performance of higher-order shear deformation beam theory, having quadratic variation for transverse displacement over the thickness, is superior in determining the SERR for multidirectional DCB specimen.
\end{abstract}

DOI: 10.1061/(ASCE)0733-9399(2009)135:10(1119)

CE Database subject headings: Composite structures; Delaminating; Cracking; Shear deformation; Toughness; Theories.

\section{Introduction}

The most commonly used test specimen for the determination of Mode I (opening mode) interlaminar fracture toughness (IFT) of laminated composites is double cantilever beam (DCB) specimen [Fig. 1(a)]. Sela and Ishai (1989) reviewed the state of the art in the subject of interlaminar fracture toughness of laminated composites covering Mode I testing using DCB specimen. Davies and Benzeggagh (1989) presented a detailed review on the interlaminar Mode I fracture testing, in particular on the DCB test specimen, covering specimen preparation, data reduction methods, and the factors affecting DCB test results. Here, only few relevant analytical research works of DCB specimen have been reported. Isotropic DCB specimen was analyzed by Benbow and Roesler (1957), Gilman (1960), and Kanninen (1973) using the Euler-Bernoulli beam theory, by Kanninen (1974) using the Timoshenko beam theory, by Chang et al. (1976) using the refined plate theory (having quadratic transverse displacement variation in the thickness coordinate) and two-dimensional elastostatic theory under plane strain assumption, by Williams (1988) using the conventional beam theory with consideration to shear deformation and by Sun and Pandey (1994) using the Timoshenko beam theory for cracked part and the approximate twodimensional elasticity solution to estimate the root rotations of the beam segments at the crack tip. Kanninen $(1973,1974)$ used the

${ }^{1}$ Professor, Civil Engineering Dept., Indian Institute of Science, Bangalore 560 012, India. E-mail: bkr@civil.iisc.ernet.in

${ }_{2}^{2}$ Scientist, Structures Div., National Aerospace Laboratories, Bangalore 560 017, India; formerly, Research Student, Civil Engineering Dept., Indian Institute of Science, Bangalore 560 012, India (corresponding author). E-mail: pavan@nal.res.in

Note. This manuscript was submitted on June 24, 2004; approved on April 21, 2009; published online on September 15, 2009. Discussion period open until March 1, 2010; separate discussions must be submitted for individual papers. This paper is part of the Journal of Engineering Mechanics, Vol. 135, No. 10, October 1, 2009. CASCE, ISSN 07339399/2009/10-1119-1128/\$25.00. concepts of beam on elastic foundation for uncracked part of isotropic DCB specimen to study the effect of crack root flexibility. Li et al. (2004) studied in detail the effect of transverse shear on SERR for delamination in layered, isotropic, linear elastic materials and applied to symmetrical DCB specimen. They showed that the "correction factor," in the resulting SERR expression of DCB specimen, arises from shear component and it does not arise from factors such as root rotation, cohesive zones, or elastic foundations. Pradeilles-Duval (2004) analyzed isotropic DCB specimen using the Kirchhoff-Love plate theory (cylindrical bending) to study the propagation of delamination in a DCB via a global energetic analysis. Unidirectional DCB specimen was analyzed by Whitney (1985) using the higher-order plate theory having linear transverse displacement variation in the thickness coordinate under cylindrical bending, by Williams (1989) through the extension of beam on elastic foundation model of Kanninen $(1973,1974)$ to estimate the end rotation correction by considering effective crack length correction for orthotropic materials, by Olsson (1992) using the shear corrected classical beam theory for the cracked part and with the consideration of Saint Venant effects and deformation of a beam on elastic foundation for the uncracked part, by Bao et al. (1992) using the SERR expression which consists of elementary beam term and a factor that best fits the finite element results, and by Bruno and Greco (2001) using the Kirchhoff and Reissner-Mindlin plate theories along with the interface model for the adhesion between the layers. Wang and Qiao (2004) developed a conventional bilayer model for the analysis of bilayer structure with a crack at the interface by modeling the uncracked region of the structure as two separate Reissner-Mindlin plates bonded perfectly along the interface which effectively captures the shear deformation in the uncracked region of the structure. This model is called as "semirigid joint" model as the effect of interface stresses on the deformations of the sub-beams was not considered. Wang and Qiao (2005) modified their conventional bilayer model (Wang and Qiao 2004), which has semirigid joint condition at the crack tip, by considering the deformations at the crack tip due to the interface stresses through 

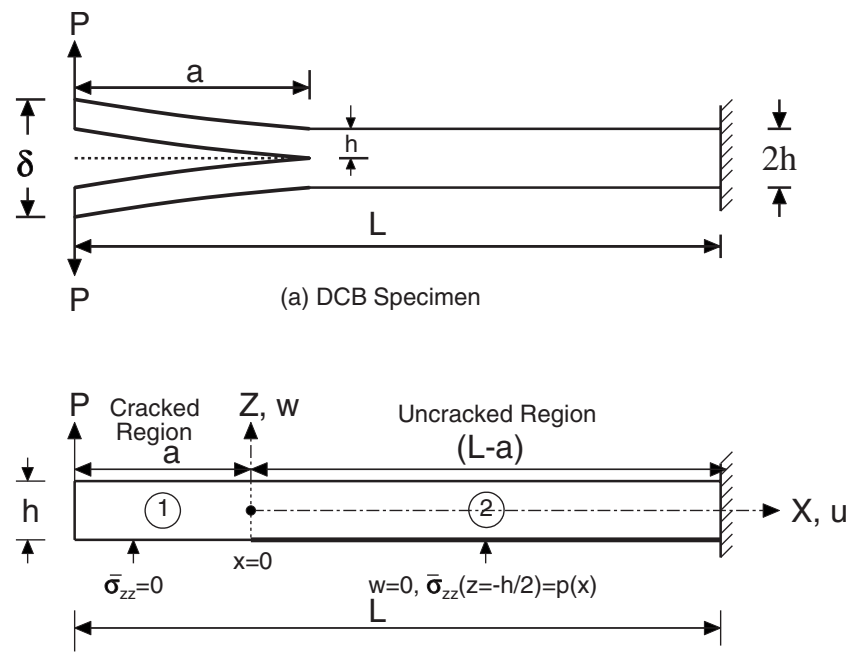

(b) Stress Analysis Model (Upper Half of the DCB Specimen)

Fig. 1. DCB specimen and its stress analysis model

the introduction of two interface compliances and called the modified model as novel bilayer model with "flexible joint" condition at the crack tip. Wang and Qiao (2005) applied this "flexible joint" model for the analysis of double cantilever specimen and obtained improved solutions for energy release rate. Composite DCB specimen was analyzed by Davidson and Schapery (1988) using the classical plate theory and by Chatterjee and Ramnath (1988) using the higher-order plate theory derived from mixed variational principle in which a laminate is treated as an assemblage of sublaminates with assumed variations of displacements and interlaminar stresses through the thickness of each sublaminate.

From the above literature and to the writers' knowledge, analysis of unidirectional and multidirectional DCB specimens using a higher-order beam theory having quadratic variation for transverse displacement over the thickness is not yet explored. Recently, to bridge this analytical gap, the writers' (Pavan Kumar and Raghu Prasad 2008) presented the mathematical modeling for the stress analysis of unidirectional DCB specimen using higherorder beam theory, having quadratic variation for transverse displacement over the thickness, by extending the stress analysis model of Whitney (1985). Here, in the present work, an attempt has been made to extend the writers' aforementioned work for symmetric multidirectional DCB specimen. Hence, the objectives of the present study are (1) mathematical modeling of stress analysis of symmetric multidirectional DCB specimen using higher-order beam theory, having quadratic variation for transverse displacement over the thickness, with variationally and nonvariationally derived matching conditions at the crack tip and (2) to study the applicability of beam theories to analyze multidirectional DCB specimen based on the fiber orientations and lamination schemes (LSs). Keeping in view the above points, in the present paper, mathematical modeling of stress analysis of symmetric multidirectional composite DCB specimen using classical beam theory, first and various higher-order shear deformation beam theories, has been presented to determine the Mode I strain energy release rate (SERR) for symmetric multidirectional composites.
Table 1. $\alpha_{i}$ Values for Various Beam Theories

\begin{tabular}{lcccc}
\hline Theory & $\alpha_{1}$ & $\alpha_{2}$ & $\alpha_{3}$ & $\alpha_{4}$ \\
\hline CBT & 0 & 0 & 0 & 0 \\
FOBT & 0 & 0 & 0 & 0 \\
SOBT & 1 & 0 & 0 & 0 \\
TOBT & 1 & 1 & 0 & 0 \\
HOBT1 & 0 & 0 & 1 & 0 \\
HOBT2 & 0 & 0 & 1 & 1 \\
\hline
\end{tabular}

\section{Higher-Order Beam Theories}

The displacement field for laminated composite beam, based on classical (CBT), first order (FOBT), second order (SOBT), and third order (TOBT) of shear deformation beam theories [similar terminology and abbreviations were earlier used by Khdeir and Reddy (1994)] having constant transverse displacement over the thickness, and higher-order shear deformation beam theories (HOBT1 and HOBT2) having linearly and quadratically varying transverse displacement over the thickness, can be expressed in an unified form using the tracers $\alpha_{i}(i=1-4)$ as

$$
\begin{gathered}
u(x, z)=u_{\circ}(x)+z \psi_{x}(x)+\alpha_{1} z^{2} \phi_{x}(x)+\alpha_{2} z^{3} \xi_{x}(x) \\
w(x, z)=w_{\circ}(x)+\alpha_{3} z \psi_{z}(x)+\alpha_{4} z^{2} \phi_{z}(x)
\end{gathered}
$$

In the above equations, $x$ and $z=$ axial and transverse coordinates. $x$-axis is assumed to lie at the midplane of the beam. The parameters $u$ and $w$ define the displacement components at any general point $(x, z)$ in the beam domain. $u_{\circ}, \psi_{x}, \phi_{x}, \xi_{x}, w_{\circ}, \psi_{z}$, and $\phi_{z}$ $=$ generalized displacements defined along the $x$-axis at the midplane $(z=0)$. For classical beam theory, $\psi_{x}=-d w_{\circ} / d x$ in Eq. (1) and in subsequent derivations. The tracers $\alpha_{i}$, appearing in Eqs. (1), are defined for various beam models as given in Table 1. Further, it may be noted that the terms, equations and relations, associated with the tracers $\alpha_{i}$, exist only if $\alpha_{i}$ is "unity" and do not exist if $\alpha_{i}$ is " 0 ."

The strain displacement relations corresponding to Eqs. (1) are

$$
\begin{gathered}
\epsilon_{x x}=u_{\circ, x}+z \psi_{x, x}+\alpha_{1} z^{2} \phi_{x, x}+\alpha_{2} z^{3} \xi_{x, x} ; \quad \epsilon_{z z}=\alpha_{3} \psi_{z}+2 \alpha_{4} z \phi_{z} \\
\gamma_{x z}=\left(w_{\circ, x}+\psi_{x}\right)+2 \alpha_{1} z \phi_{x}+3 \alpha_{2} z^{2} \xi_{x}+\alpha_{3} z \psi_{z, x}+\alpha_{4} z^{2} \phi_{z, x}
\end{gathered}
$$

in which ( $)_{, x}$ represents differentiation with respect to " $x$."

Based on the displacement field [Eq. (1)], strain displacement relations [Eq. (2)], and stress-strain relations [Eq. (24) and (25)] given in the Appendix and using the principle of minimum potential energy and variational calculus, the equilibrium equations for a laminated composite beam, having width " $b$ " and thickness " $h$," subjected to external surface traction $\bar{\sigma}_{z z}=p(x)$ at the bottom surface $(z=-h / 2)$, can be written as (Pavan Kumar and Raghu Prasad 2008)

$$
\begin{gathered}
N_{x x, x}=0 ; \quad Q_{x z, x}-b p=0 ; \quad M_{x x, x}-Q_{x z}=0 \\
\alpha_{1}\left(S_{x x, x}-2 R_{x z}\right)=0 ; \quad \alpha_{2}\left(P_{x x, x}-3 T_{x z}\right)=0 \\
\alpha_{3}\left[R_{x z, x}-N_{z z}+(b h / 2) p\right]=0 ; \quad \alpha_{4}\left[T_{x z, x}-2 M_{z z}-\left(b h^{2} / 4\right) p\right]=0
\end{gathered}
$$

In the above equations, $N_{x x}, M_{x x}, S_{x x}, P_{x x}, N_{z z}, M_{z z}, Q_{x z}, R_{x z}$, and $T_{x z}=$ stress resultants and they are defined as 


$$
\begin{gathered}
{\left[N_{x x}, M_{x x}, S_{x x}, P_{x x}\right]=b \sum_{k=1}^{n} \int_{t_{k-1}}^{t_{k}} \sigma_{x x}\left[1, z, z^{2}, z^{3}\right] d z} \\
{\left[Q_{x z}, R_{x z}, T_{x z}, N_{z z}, M_{z z}\right]=b \sum_{k=1}^{n} \int_{t_{k-1}}^{t_{k}}\left[\tau_{x z}\left(1, z, z^{2}\right), \sigma_{z z}(1, z)\right] d z}
\end{gathered}
$$

The above stress resultants can be written in terms of displacements as given below from Eqs. (4), (25), (24), (2), and (1):

In-plane stress resultants for HOBT1 and HOBT2

$$
\begin{aligned}
& N_{x x}=\widetilde{A}_{11} u_{\circ, x}+\widetilde{B}_{11} \psi_{x, x}+\alpha_{3} A_{13} \psi_{z}+2 \alpha_{4} B_{13} \phi_{z} \\
& M_{x x}=\widetilde{B}_{11} u_{\circ, x}+\widetilde{D}_{11} \psi_{x, x}+\alpha_{3} B_{13} \psi_{z}+2 \alpha_{4} D_{13} \phi_{z}
\end{aligned}
$$

In-plane stress resultants for CBT, FOBT, SOBT, and TOBT

$$
\begin{gathered}
N_{x x}=A_{11} u_{\circ, x}+B_{11} \psi_{x, x}+\alpha_{1} D_{11} \phi_{x, x}+\alpha_{2} F_{11} \xi_{x, x} \\
M_{x x}=B_{11} u_{\circ, x}+D_{11} \psi_{x, x}+\alpha_{1} F_{11} \phi_{x, x}+\alpha_{2} H_{11} \xi_{x, x} \\
S_{x x}=\alpha_{1}\left(D_{11} u_{\circ, x}+F_{11} \psi_{x, x}+H_{11} \phi_{x, x}+\alpha_{2} I_{11} \xi_{x, x}\right) \\
P_{x x}=\alpha_{2}\left(F_{11} u_{\circ, x}+H_{11} \psi_{x, x}+I_{11} \phi_{x, x}+J_{11} \xi_{x, x}\right)
\end{gathered}
$$

Interlaminar shear stress resultants for CBT, FOBT, SOBT, TOBT, HOBT1, and HOBT2

$$
\begin{aligned}
Q_{x z}=A_{55}\left(w_{\circ, x}+\psi_{x}\right)+\left(2 \alpha_{1} \phi_{x}+\alpha_{3} \psi_{z, x}\right) B_{55}+\left(3 \alpha_{2} \xi_{x}+\alpha_{4} \phi_{z, x}\right) D_{55} \\
R_{x z}=\left(\alpha_{1}+\alpha_{3}\right) B_{55}\left(w_{\circ, x}+\psi_{x}\right)+\left(2 \alpha_{1} \phi_{x}+\alpha_{3} \psi_{z, x}\right) D_{55} \\
+\left(3 \alpha_{2} \xi_{x}+\alpha_{4} \phi_{z, x}\right) F_{55} \\
T_{x z}=\left(\alpha_{2}+\alpha_{4}\right) D_{55}\left(w_{\circ, x}+\psi_{x}\right)+\left(2 \alpha_{2} \phi_{x}+\alpha_{4} \psi_{z, x}\right) F_{55} \\
+\left(3 \alpha_{2} \xi_{x}+\alpha_{4} \phi_{z, x}\right) H_{55}
\end{aligned}
$$

Interlaminar normal stress resultants for HOBT1 and HOBT2

$$
\begin{gathered}
N_{z z}=\alpha_{3}\left(A_{13} u_{\circ, x}+B_{13} \psi_{x, x}+A_{33} \psi_{z}+2 \alpha_{4} B_{33} \phi_{z}\right) \\
M_{z z}=\alpha_{4}\left(B_{13} u_{\circ, x}+D_{13} \psi_{x, x}+B_{33} \psi_{z}+2 D_{33} \phi_{z}\right)
\end{gathered}
$$

The quantities $\widetilde{A}_{11}, \widetilde{B}_{11}, \widetilde{D}_{11}, A_{11}, B_{11}, D_{11}, F_{11}, H_{11}, I_{11}, J_{11}, A_{13}$, $B_{13}, D_{13}, A_{33}, B_{33}, D_{33}, A_{55}, B_{55}, D_{55}, F_{55}$, and $H_{55}$, appearing in Eqs. (5)-(8), are defined as

$$
\begin{gathered}
{\left[\widetilde{A}_{11}, \widetilde{B}_{11}, \widetilde{D}_{11}\right]=b \sum_{k=1}^{n} \int_{t_{k-1}}^{t_{k}} Q_{11}\left[1, z, z^{2}\right] d z} \\
{\left[A_{11}, B_{11}, D_{11}, F_{11}, H_{11}, I_{11}, J_{11}\right]=b \sum_{k=1}^{n} \int_{t_{k-1}}^{t_{k}} Q_{11}^{r}\left[1, z, z^{2}, z^{3}, z^{4}, z^{5}, z^{6}\right] d z} \\
{\left[A_{13}, B_{13}, D_{13}, A_{33}, B_{33}, D_{33}\right]=b \sum_{k=1}^{n} \int_{t_{k-1}}^{t_{k}}\left[Q_{13}\left(1, z, z^{2}\right), Q_{33}\left(1, z, z^{2}\right)\right] d z} \\
{\left[A_{55}, B_{55}, D_{55}, F_{55}, H_{55}\right]=b \sum_{k=1}^{n} \int_{t_{k-1}}^{t_{k}} k Q_{55}\left[1, z, z^{2}, z^{3}, z^{4}\right] d z}
\end{gathered}
$$

where " $k$ " = shear correction factor and has been used only for FOBT, HOBT1, and HOBT2 beam theories. Now, it is possible to have two choices for the interlaminar shear stress resultant expressions of FOBT, HOBT1, and HOBT2. The first choice is without the shear correction factor (i.e., $k=1$ ). The second choice is with the assumed shear correction factor " $k=5 / 6$ " and the beam theories have been named as $\mathrm{FOBT}^{k}, \mathrm{HOBT}^{k}$, and $\mathrm{HOBT}^{k}$ (superscript " $k$ " indicates that the shear correction factor has been used).

\section{Mathematical Modeling of DCB Stress Analysis Model}

Figs. 1(a and b) show the DCB specimen and its stress analysis model, respectively. The stress analysis model is similar to the stress analysis model proposed by Whitney (1985). The stress analysis model considers only upper half (above delamination plane) of the DCB specimen because of the fact that the delamination is at midplane and LS and loading are symmetric about the midplane of the DCB specimen. Further, the stress analysis model has cracked $[-a \leqslant x \leqslant 0]$ and uncracked $[0 \leqslant x \leqslant(L-a)]$ regions and are idealized as two individual Beam 1 and Beam 2, respectively, with an imaginary cut at the crack tip. The cracked region is free of all surface tractions. But, in the uncracked region, at the bottom of the stress analysis model (i.e., at the midplane of the DCB specimen), the surface traction " $\bar{\sigma}_{z z}=p(x)$ " exists and the vertical displacement is 0 (i.e., $w=0$ ). The governing differential equations for cracked and uncracked regions, based on the beam theories considered, have been presented in the next subsection.

\section{Governing Differential Equations for Cracked and Uncracked Regions}

The cracked and uncracked regions of the DCB stress analysis model can be treated as laminated composite beam without surface tractions and with surface traction " $p(x)$ " at the bottom surface, respectively. Hence, the equilibrium equations for cracked and uncracked regions can be obtained from Eqs. (3) without and with surface traction " $p(x)$," respectively.

\section{Modifications for HOBT1 and HOBT2}

While applying HOBT1 and HOBT2 to analyze DCB specimen, following Whitney (1985), a further assumption has been made that the through-the-thickness stress $\sigma_{z z}$ has negligible effect on the in-plane stress $\sigma_{x x}$. Thus, a plane stress state (in the thickness direction) has been assumed relative to in-plane stress resultants. Due to this assumption, first part of the Eqs. (24) is modified for HOBT1 and HOBT2 as

$$
\sigma_{x x}=Q_{11}^{r} \epsilon_{x x}
$$

which is similar to the first part of Eqs. (25) obtained under the plane stress state condition in the thickness direction as defined in the Appendix. Because of the modified $\sigma_{x x}$, the in-plane stress resultants $N_{x x}$ and $M_{x x}$ of Eqs. (5) will be modified for HOBT1 and HOBT2 as

$$
N_{x x}=A_{11} u_{\circ, x}+B_{11} \psi_{x, x} ; M_{x x}=B_{11} u_{\circ, x}+D_{11} \psi_{x, x}
$$

which are similar to the $N_{x x}$ and $M_{x x}$ expressions of FOBT.

\section{Cracked Region $[-a \leqslant x \leqslant 0]$}

The governing differential equations for cracked region in terms of generalized displacements can be obtained by using Eqs. (6) -(8) and (11) in Eqs. (3) with $p(x)=0$ and they can be written in an unified form for various beam theories as given below

$$
A_{11} u_{\circ, x x}+B_{11} \psi_{x, x x}+\alpha_{1} D_{11} \phi_{x, x x}+\alpha_{2} F_{11} \xi_{x, x x}=0
$$




$$
\begin{gathered}
A_{55}\left(w_{\circ, x x}+\psi_{x, x}\right)+\left(2 \alpha_{1} \phi_{x, x}+\alpha_{3} \psi_{z, x x}\right) B_{55}+\left(3 \alpha_{2} \xi_{x, x}+\alpha_{4} \phi_{z, x x}\right) D_{55} \\
=0 \\
B_{11} u_{\circ, x x}+D_{11} \psi_{x, x x}+\alpha_{1} F_{11} \phi_{x, x x}+\alpha_{2} H_{11} \xi_{x, x x}-A_{55}\left(w_{\circ, x}+\psi_{x}\right) \\
-\left(2 \alpha_{1} \phi_{x}+\alpha_{3} \psi_{z, x}\right) B_{55}-\left(3 \alpha_{2} \xi_{x}+\alpha_{4} \phi_{z, x}\right) D_{55}=0 \\
\alpha_{1}\left[D_{11} u_{\circ, x x}+F_{11} \psi_{x, x x}+H_{11} \phi_{x, x x}+\alpha_{2} I_{11} \xi_{x, x x}\right. \\
\left.-2 B_{55}\left(w_{\circ, x}+\psi_{x}\right)-4 D_{55} \phi_{x}-6 \alpha_{2} F_{55} \xi_{x}\right]=0 \\
\alpha_{2}\left[F_{11} u_{\circ, x x}+H_{11} \psi_{x, x x}+I_{11} \phi_{x, x x}+J_{11} \xi_{x, x x}\right. \\
\left.-3 D_{55}\left(w_{\circ, x}+\psi_{x}\right)-6 F_{55} \phi_{x}-9 H_{55} \xi_{x}\right]=0 \\
\alpha_{3}\left[B_{55}\left(w_{\circ, x}+\psi_{x}\right)+D_{55} \psi_{z, x}+\alpha_{4} F_{55} \phi_{z, x}\right. \\
\left.-A_{13} u_{\circ, x}-B_{13} \psi_{x, x}-A_{33} \psi_{z}-2 \alpha_{4} B_{33} \phi_{z}\right]=0 \\
\alpha_{4}\left[D_{55}\left(w_{\circ, x}+\psi_{x}\right)+F_{55} \psi_{z, x}+H_{55} \phi_{z, x}\right. \\
\left.-2 B_{13} u_{\circ, x}-2 D_{13} \psi_{x, x}-2 B_{33} \psi_{z}-4 D_{33} \phi_{z}\right]=0
\end{gathered}
$$

\section{Uncracked Region $[0 \leqslant x \leqslant(L-a)]$}

In the uncracked region, $p(x) \neq 0$. Further, the transverse displacement " $w$ " is 0 at the bottom surface $(z=-h / 2)$ of the stress analysis model. This can be written as

$$
w(z=-h / 2)=0
$$

By using the second part of Eqs. (1) in the above Eq. (13), w。 can be expressed in terms of $\psi_{z}$ and $\phi_{z}$ as

$$
w_{\circ}=\alpha_{3}(h / 2) \psi_{z}-\alpha_{4}\left(h^{2} / 4\right) \phi_{z}
$$

and now the transverse normal traction " $p(x)$ " is the unknown. Consequently, by using Eq. (14) in Eqs. (7), the interlaminar shear stress resultant expressions of $Q_{x z}, R_{x z}$, and $T_{x z}$ will be modified as

$$
\begin{aligned}
Q_{x z}= & A_{55} \psi_{x}+2 \alpha_{1} B_{55} \phi_{x}+3 \alpha_{2} D_{55} \xi_{x}+\alpha_{3}\left\{\left(A_{55} / 2\right) h+B_{55}\right\} \psi_{z, x} \\
+ & \alpha_{4}\left\{D_{55}-\left(A_{55} / 4\right) h^{2}\right\} \phi_{z, x} \\
R_{x z}= & \left(\alpha_{1}+\alpha_{3}\right) B_{55} \psi_{x}+2 \alpha_{1} D_{55} \phi_{x}+3 \alpha_{2} F_{55} \xi_{x} \\
& +\alpha_{3}\left\{\left(B_{55} / 2\right) h+D_{55}\right\} \psi_{z, x}+\alpha_{4}\left\{F_{55}-\left(B_{55} / 4\right) h^{2}\right\} \phi_{z, x} \\
T_{x z}= & \left(\alpha_{2}+\alpha_{4}\right) D_{55} \psi_{x}+\alpha_{2}\left(2 F_{55} \phi_{x}+3 H_{55} \xi_{x}\right) \\
& +\alpha_{4}\left[\left\{\left(D_{55} / 2\right) h+F_{55}\right\} \psi_{z, x}+\left\{H_{55}-\left(D_{55} / 4\right) h^{2}\right\} \phi_{z, x}\right]
\end{aligned}
$$

By using Eqs. (6)-(8), (11), and (15) in Eqs. (3), the following differential equations for the uncracked region, in terms of generalized displacements and surface traction " $p(x)$," can be written in an unified form for various beam theories:

$$
\begin{gathered}
A_{11} u_{\circ, x x}+B_{11} \psi_{x, x x}+\alpha_{1} D_{11} \phi_{x, x x}+\alpha_{2} F_{11} \xi_{x, x x}=0 \\
A_{55} \psi_{x, x}+2 \alpha_{1} B_{55} \phi_{x, x}+3 \alpha_{2} D_{55} \xi_{x, x}+\alpha_{3}\left\{\left(A_{55} / 2\right) h+B_{55}\right\} \psi_{z, x x} \\
+\alpha_{4}\left\{D_{55}-\left(A_{55} / 4\right) h^{2}\right\} \phi_{z, x x}-b p=0 \\
B_{11} u_{\circ, x x}+D_{11} \psi_{x, x x}+\alpha_{1} F_{11} \phi_{x, x x}+\alpha_{2} H_{11} \xi_{x, x x}-A_{55} \psi_{x}-2 \alpha_{1} B_{55} \phi_{x} \\
-3 \alpha_{2} D_{55} \xi_{x}-\alpha_{3}\left\{\left(A_{55} / 2\right) h+B_{55}\right\} \psi_{z, x} \\
-\alpha_{4}\left\{D_{55}-\left(A_{55} / 4\right) h^{2}\right\} \phi_{z, x}=0
\end{gathered}
$$

$$
\begin{gathered}
\alpha_{1}\left[D_{11} u_{\circ, x x}+F_{11} \psi_{x, x x}+H_{11} \phi_{x, x x}+\alpha_{2} I_{11} \xi_{x, x x}\right. \\
\left.-2 B_{55} \psi_{x}-4 D_{55} \phi_{x}-6 \alpha_{2} F_{55} \xi_{x}\right]=0 \\
\alpha_{2}\left[F_{11} u_{\circ, x x}+H_{11} \psi_{x, x x}+I_{11} \phi_{x, x x}+J_{11} \xi_{x, x x}\right. \\
\left.-3 D_{55} \psi_{x}-6 F_{55} \phi_{x}-9 H_{55} \xi_{x}\right]=0
\end{gathered}
$$$$
\alpha_{3}\left[B_{55} \psi_{x, x}+\left\{\left(B_{55} / 2\right) h+D_{55}\right\} \psi_{z, x x}+\alpha_{4}\left\{F_{55}-\left(B_{55} / 4\right) h^{2}\right\} \phi_{z, x x}\right.
$$$$
\left.-A_{13} u_{\circ, x}-B_{13} \psi_{x, x}-A_{33} \psi_{z}-2 \alpha_{4} B_{33} \phi_{z}+(b h / 2) p\right]=0
$$$$
\alpha_{4}\left[D_{55} \psi_{x, x}+\left\{\left(D_{55} / 2\right) h+F_{55}\right\} \psi_{z, x x}+\left\{H_{55}-\left(D_{55} / 4\right) h^{2}\right\} \phi_{z, x x}\right.
$$$$
\left.-2 B_{13} u_{\circ, x}-2 D_{13} \psi_{x, x}-2 B_{33} \psi_{z}-4 D_{33} \phi_{z}-\left(b h^{2} / 4\right) p\right]=0
$$

Solution to Eqs. (12) and (16), respectively for cracked and uncracked regions, is straight forward and can be easily obtained. The solution details are omitted here for the sake of brevity and are available elsewhere (Pavan Kumar 1999). However, it may be worth mentioning here that in the process of writing solutions to Eqs. (12) and (16), one will come across second, fourth, and sixth order differential equations whose auxiliary equations can have real roots or complex roots or combination of real and complex roots. Here, for the material properties and LSs considered, all the roots are found to be real. The solution of Eqs. (12) and (16) consists of $18(10+8)$ [10 constants for cracked region and eight constants for uncracked region], $14(8+6), 18(10+8)$, $14(8+6), 10(6+4)$, and $8(6+2)$ integration or arbitrary constants for HOBT2, HOBT1, TOBT, SOBT, FOBT, and CBT, respectively. These arbitrary constants can be determined from the boundary conditions at the edges and matching conditions at the crack tip of DCB specimen, which are given in the next subsection.

\section{Boundary and Matching Conditions for DCB Stress Analysis Model}

Recently, Pavan Kumar and Raghu Prasad (2008) have derived the boundary conditions at the edges and matching conditions at the crack tip for DCB stress analysis model and they are as given below:

At the free end $[x=-a]$

$$
\begin{gathered}
N_{x x}^{(1)}=0 ; Q_{x z}^{(1)}=-P ; M_{x x}^{(1)}=0 ; \alpha_{1} S_{x x}^{(1)}=0 \\
\alpha_{2} P_{x x}^{(1)}=0 ; \quad \alpha_{3} R_{x z}^{(1)}=0 ; \quad \alpha_{4} T_{x z}^{(1)}=0
\end{gathered}
$$

At the crack tip $[x=0]$

$$
u_{\circ}^{(1)}=u_{\circ}^{(2)} ; w_{\circ}^{(1)}=\left\{w_{\circ}^{(2)}=\left(\alpha_{3} \frac{h}{2} \psi_{z}^{(2)}-\alpha_{4} \frac{h^{2}}{4} \phi_{z}^{(2)}\right)\right\}
$$

$\psi_{x}^{(1)}=\psi_{x}^{(2)}($ except for CBT $) ; w_{\circ, x}^{(1)}=\left(w_{\circ, x}^{(2)}=0\right)($ only for CBT $)$

$$
\begin{gathered}
\alpha_{1} \phi_{x}^{(1)}=\alpha_{1} \phi_{x}^{(2)} ; \quad \alpha_{2} \xi_{x}^{(1)}=\alpha_{2} \xi_{x}^{(2)} ; \\
\alpha_{3} \psi_{z}^{(1)}=\alpha_{3} \psi_{z}^{(2)} ; \quad \alpha_{4} \phi_{z}^{(1)}=\alpha_{4} \phi_{z}^{(2)} \\
N_{x x}^{(1)}=N_{x x}^{(2)} ; M_{x x}^{(1)}=M_{x x}^{(2)}(\text { except for CBT }) \\
\alpha_{1} S_{x x}^{(1)}=\alpha_{1} S_{x x}^{(2)} ; \quad \alpha_{2} P_{x x}^{(1)}=\alpha_{2} P_{x x}^{(2)}
\end{gathered}
$$




$$
\begin{aligned}
& \alpha_{3}\left(R_{x z}^{(1)}+\frac{h}{2} Q_{x z}^{(1)}\right)=\alpha_{3}\left(R_{x z}^{(2)}+\frac{h}{2} Q_{x z}^{(2)}\right) \\
& \alpha_{4}\left(T_{x z}^{(1)}-\frac{h^{2}}{4} Q_{x z}^{(1)}\right)=\alpha_{4}\left(T_{x z}^{(2)}-\frac{h^{2}}{4} Q_{x z}^{(2)}\right)
\end{aligned}
$$

At the clamped support $[x=(L-a)]$

$$
\begin{gathered}
u_{\circ}^{(2)}=0 ; \quad \psi_{x}^{(2)}=0(\text { except for CBT }) \\
\alpha_{1} \phi_{x}^{(2)}=0 ; \quad \alpha_{2} \xi_{x}^{(2)}=0 ; \quad \alpha_{3} \psi_{z}^{(2)}=0 ; \alpha_{4} \phi_{z}^{(2)}=0
\end{gathered}
$$

Special attention has been given for the matching conditions at the crack tip and they have been derived by enforcing the displacement continuity at the crack tip in conjunction with the variational equation. It may be noted that the matching conditions at the crack tip will integrate the cracked and uncracked regions. From Eqs. (17)-(19), it can be noted that HOBT2, HOBT1, TOBT, SOBT, FOBT, and CBT will have 18, 14, 18, 14, 10, and 8 boundary and matching conditions, respectively. Application of the above boundary and matching conditions [Eqs. (17)-(19)] results in a system of simultaneous equations, which can be solved numerically using the standard routines of gauss elimination method for arbitrary constants. After obtaining the arbitrary constants, the deflection under the load and compliance can be calculated and then the SERR can be determined using compliance approach.

Recently, Pavan Kumar and Raghu Prasad (2008) found that, in the case of HOBT1 and HOBT2 beam theories, the application of nonvariationally derived matching conditions at the crack tip showed better performance than the application of variationally derived matching conditions at the crack tip in determining the SERR for unidirectional DCB specimen in comparison with existing literature reported by Olsson (1992). Hence, in the present work of SERR solution for multidirectional DCB specimen also, an attempt has been made to apply the nonvariationally derived matching conditions at the crack tip for HOBT1 and HOBT2 models of DCB specimen. In the case of HOBT1 model, instead of the 13th part of Eqs. (18), continuity condition for $Q_{x z}$ (i.e., $\left.Q_{x z}^{(1)}=Q_{x z}^{(2)}\right)$ at the crack tip has been imposed and $R_{x z}$ has been omitted from the matching conditions and the corresponding model is called as $\operatorname{HOBT}_{Q}^{k}$. Similarly, in the case of HOBT2 model, instead of the 13th and 14th parts of Eqs. (18), continuity conditions for $Q_{x z}$ (i.e., $Q_{x z}^{(1)}=Q_{x z}^{(2)}$ ) and $T_{x z}$ (i.e., $T_{x z}^{(1)}=T_{x z}^{(2)}$ ) at the crack tip have been imposed and $R_{x z}$ has been omitted from the matching conditions and the corresponding model is called as $\operatorname{HOBT} 2_{Q T}^{k}$. It may be noted here that the DCB specimen model of Whitney (1985) is same as the present HOBT1 model of DCB specimen with nonvariationally derived matching conditions at the crack tip $\left(\operatorname{HOBT} 1_{Q}^{k}\right)$.

\section{Compliance and SERR}

The compliance " $C$ " can be obtained from the following relation:

$$
C=\delta / P
$$

where " $\delta=2 w^{(1)}(-a, h / 2) "=$ deflection under the load for DCB specimen.

The SERR and compliance are related by the following formula (Broek 1982):

$$
G_{I}=\left[P^{2} /(2 b)\right](d C / d a)
$$

It may be noted here that $(d C / d a)$ has not been determined explicitly as it is tedious particularly in the case of beam theories SOBT, TOBT, HOBT1, and HOBT2. Therefore, the derivative $(d C / d a)$ has been obtained using the finite difference method by adopting the central finite difference approximation. Then the compliance derivative can be written as

$$
(d C / d a)_{n}=\left(C_{n+1}-C_{n-1}\right) /\left(2 \Delta_{s}\right)
$$

in which $(d C / d a)_{n}=$ compliance derivative at the delamination length $a_{n} ; C_{n+1}=$ compliance when the delamination length is $a_{n+1}$; $C_{n-1}=$ compliance when the delamination length is $a_{n-1}$; and $\Delta_{s}$ $=$ spacing between two successive delamination lengths. Hence, we can obtain SERR from the following expression:

$$
G_{I I}=\left[P^{2} /(2 b)\right]\left[\left(C_{n+1}-C_{n-1}\right) /\left(2 \Delta_{s}\right)\right]
$$

The FORTRAN programs have been written for all the required mathematical steps to analyze multidirectional DCB specimen using the beam theories CBT, FOBT, SOBT, TOBT, HOBT1, and HOBT2.

\section{Results and Discussion}

\section{Comparison with Earlier Research}

First, to validate the present DCB stress analysis model formulation and to study the applicability of beam theories (classical and shear deformation) for the SERR solution of composite DCB specimen in the presence of unidirectional, cross-ply and multidirectional composite laminates, the SERR values from the various beam models of present work have been compared with the results available in the literature.

Table 2 shows the comparison of SERR values from the various beam models of present work with the SERR values reported by Schön et al. (2000) for unidirectional $\left(\left[0_{12}^{\circ}\right]_{s}\right)$ and crossply $\left(\left[\left(90^{\circ} / 0^{\circ} / 90^{\circ} / 0^{\circ} / 90^{\circ} / 0^{\circ}\right)_{s}\right]_{s}\right)$ laminated composite DCB specimens and Davidson et al. (1996) for multidirectional $\left(\left[ \pm 30 \% 0_{3}^{\circ} /-30 \% / 0 \% 30_{2}^{\circ} / 0 \%-30 \% 0_{3}^{\circ} / \mp 30^{\circ}\right]_{s}\right)$ composite DCB specimen. The LSs $\left[0_{12}^{\circ}\right]_{s},\left[\left(90^{\circ} / 0^{\circ} / 90^{\circ} / 0^{\circ} / 90^{\circ} / 0^{\circ}\right)_{s}\right]_{s}$ and $\left[ \pm 30^{\circ} / 0_{3}^{\circ} /-30^{\circ} / 0^{\circ} / 30_{2}^{\circ} / 0^{\circ} /-30^{\circ} / 0_{3}^{\circ} / \mp 30^{\circ}\right]_{s}$ are referred to as LS-1, LS-2, and LS-3, respectively. For the unidirectional and cross-ply composite DCB specimens, the material properties (IM7/8552 Graphite/Epoxy) are $E_{11}=160 \mathrm{GPa}, E_{22}=10 \mathrm{GPa}, E_{33}$ $=10 \mathrm{GPa}, G_{12}=4.8 \mathrm{GPa}, G_{13}=4.8 \mathrm{GPa}, G_{23}=3.2 \mathrm{GPa}, v_{12}=0.31$, $v_{13}=0.31$, and $v_{32}=v_{23}=0.52$. The geometrical dimensions are length $(L)=205 \mathrm{~mm}$, thickness $(2 h)=3.12 \mathrm{~mm}$, width $(b)$ $=20 \mathrm{~mm}$, and crack length $(a)=50 \mathrm{~mm}$. The loading is $P$ $=100 \mathrm{~N}$. Next, for the multidirectional composite DCB specimen, the material properties (Ciba-Geigy C12K/R6376 Graphite/ Epoxy), are $E_{11}=146.86 \mathrm{GPa}, E_{22}=10.62 \mathrm{GPa}, E_{33}=10.62 \mathrm{GPa}$, $G_{12}=5.45 \mathrm{GPa}, G_{13}=5.45 \mathrm{GPa}, G_{23}=3.99 \mathrm{GPa}, v_{12}=0.33, v_{13}$ $=0.33$, and $v_{23}=0.33$. The geometrical dimensions are $L$ $=150 \mathrm{~mm}, 2 h=4.064 \mathrm{~mm}, b=25.4 \mathrm{~mm}$, and $a=57.15 \mathrm{~mm}$. The loading is assumed as $P=100 \mathrm{~N}$. The SERR values from the shear deformation beam models, which are better than those from CBT, are in good agreement with the SERR values reported by Schön et al. (2000) using finite-element analysis (FEA) for both unidirectional and cross-ply composite DCB specimens. Hence, shear deformation beam theories are important to analyze composite DCB specimens to obtain the SERR accurately. Shear deformation beam theories are better than CBT due to the consideration of 
Table 2. Comparison of SERR Values from the Present Work with Those from Schön et al. (2000) for Unidirectional and Cross-Ply Composite DCB Specimens and Davidson et al. (1996) for Multidirectional Composite DCB Specimen

\begin{tabular}{|c|c|c|c|c|c|c|}
\hline \multirow[b]{2}{*}{ Model/theory } & \multicolumn{6}{|c|}{ Strain energy release rate $\left(\mathrm{kJ} / \mathrm{m}^{2}\right)$} \\
\hline & \multicolumn{2}{|c|}{ LS-1 } & \multicolumn{2}{|c|}{ LS-2 } & \multicolumn{2}{|c|}{ LS-3 } \\
\hline Schön et al. $(2000)^{\mathrm{a}}$ & \multicolumn{2}{|c|}{1.3922} & \multicolumn{2}{|c|}{3.2560} & \multicolumn{2}{|c|}{-} \\
\hline Davidson et al. (1996) ${ }^{\mathrm{a}}$ & \multicolumn{2}{|c|}{ - } & \multicolumn{2}{|c|}{ - } & \multicolumn{2}{|c|}{0.8025} \\
\hline Davidson et al. $(1996)^{\mathrm{b}}$ & \multicolumn{2}{|c|}{-} & \multicolumn{2}{|c|}{-} & \multicolumn{2}{|c|}{0.7930} \\
\hline Present work & pl. $\epsilon$ & pl. $\sigma$ & pl. $\epsilon$ & pl. $\sigma$ & pl. $\epsilon$ & pl. $\sigma$ \\
\hline $\mathrm{CBT}$ & 1.2273 & 1.2347 & 2.9640 & 2.9820 & 0.6476 & 1.0273 \\
\hline FOBT/SOBT & 1.3587 & 1.3665 & 3.1860 & 3.2046 & 0.7103 & 1.1062 \\
\hline TOBT & 1.3688 & 1.3766 & 3.2103 & 3.2289 & 0.7162 & 1.1181 \\
\hline HOBT1 & 1.3706 & 1.3803 & 3.2114 & 3.2319 & 0.7180 & 1.1184 \\
\hline HOBT2 & 1.3742 & 1.3849 & 3.2182 & 3.2396 & 0.7203 & 1.1224 \\
\hline $\mathrm{FOBT}^{k}$ & 1.3716 & 1.3794 & 3.2076 & 3.2262 & 0.7164 & 1.1139 \\
\hline HOBT $1^{k}$ & 1.3827 & 1.3924 & 3.2314 & 3.2520 & 0.7237 & 1.1254 \\
\hline $\mathrm{HOBT}^{k}$ & 1.3861 & 1.3967 & 3.2378 & 3.2591 & 0.7259 & 1.1292 \\
\hline $\mathrm{HOBT1}_{Q}^{k c}$ & 1.3953 & 1.4012 & 3.2580 & 3.2810 & 0.7321 & 1.1394 \\
\hline $\mathrm{HOBT} 2_{O T}^{k} \mathrm{c}$ & 1.3987 & 1.4116 & 3.2644 & 3.2881 & 0.7343 & 1.1432 \\
\hline
\end{tabular}

${ }^{\mathrm{a}}$ Finite-element analysis.

${ }^{\mathrm{b}}$ Classical plate theory under generalized plane stress condition.

${ }^{\mathrm{c}}$ Nonvariationally derived matching conditions have been applied at the crack tip.

shear deformation effects in their formulation. It can also be seen that the difference between the plane strain and plane stress analyses is marginal for unidirectional and cross-ply composite laminates as the $E_{11} / E_{22}$ ratio is 16 , which is sufficiently large (Pavan Kumar and Raghu Prasad 2003). In the case of multidirectional DCB specimens, the SERR values from various beam models under plane strain (Pl. $\epsilon$ ) condition are agreeing moderately with those from FEA of Davidson et al. (1996), where as the SERR values from various beam models under plane stress (Pl.o) condition do not agree with those from FEA of Davidson et al. (1996). This may be due to the reason that FEA used for comparison is based on three-dimensional (3D) finite elements, width of the specimen larger than its thickness, and the presence of multidirectional composite laminates. Further, it may be noted from Davidson et al. (1996) that the SERR values from the classical laminated plate theory (CPT) under "generalized Pl. $\sigma$ condition," where generalized forces in the width direction are set to 0 , are in good agreement with those from FEA. Hence, the present DCB stress analysis model concepts can be extended in conjunction with the higher-order laminated plate theories under "generalized Pl. $\sigma$ condition" to determine the SERR accurately for symmetric multidirectional composites. Further, it can be said that the SERR values from shear deformation beam models (Pl.є) are in moderate agreement with the FEA SERR values and better than those from CBT (Pl.€). It may also be observed that, for symmetric laminated composites, FOBT and SOBT give similar results. This is due to the reason that the solution results in $\phi_{x}$ $=0$ for symmetric laminated composites in the case of SOBT.

Based on the comparative study shown in Table 2 between the present work and available literature, it can be said that the beam theories under both Pl. $\sigma$ and Pl.€ conditions in the width direction are applicable for unidirectional and cross-ply composite DCB specimens with good performance. Further, the beam theories are applicable only under Pl. $\epsilon$ condition in the width direction for multidirectional DCB specimens with moderate performance. Among the shear deformation theories considered, performances of DCB specimen models based on HOBT1 and HOBT2 theories are good in determining the SERR for unidirectional, cross-ply, and multidirectional DCB specimens. Also, in a separate study (Pavan Kumar and Raghu Prasad 2008), the writers observed that the HOBT2 model with nonvariationally derived matching conditions at the crack tip $\left(\mathrm{HOBT}_{Q T}^{k}\right)$ gives good performance in comparison with the earlier research works of Whitney (1985), Crews et al. (1991) (using FEA), and Olsson (1992) in determining the SERR for unidirectional DCB specimen. The DCB specimen examples presented in Table 2 have a large $a / h$ ratio. Hence, to show the high accuracy of the present work based on the HOBT2 theory for a small $a / h$ ratio, here we reproduce the results from writers' aforementioned work, which is a special case of the present work, along with the additional comparison with Bao et al. (1992) and Wang and Qiao (2005) for unidirectional DCB specimen having various crack lengths in Table 3 . The material properties for DCB specimen are $E_{11}=134 \mathrm{GPa}, E_{22}=E_{33}$ $=13 \mathrm{GPa}, G_{13}=6.4 \mathrm{GPa}$ and $v_{12}=0.3$. The geometrical dimensions of DCB specimen are half thickness $(h)=1.65 \mathrm{~mm}$ and width $(b)=25.4 \mathrm{~mm}$. The loading is $P=0.0254 \mathrm{~N}$. It may be interesting to observe from Table 3 that the SERR values from HOBT $2^{k}$ model (with variationally derived matching conditions at the crack tip) are in good agreement with the SERR values from both Bao et al. (1992) and Wang and Qiao (2005) for all the crack lengths including small $a / h$ ratios. The good agreement, between HOBT $2^{k}$ model and simple novel bilayer model of Wang and Qiao (2005), may be due to the reason that HOBT2 theory uses interlaminar normal stress " $\sigma_{z z}$ " and transverse shear stress " $\tau_{x z}$ " in the constitutive equations along with the variationally derived matching conditions at the crack tip of DCB specimen and Wang and Qiao (2005) considers the flexible joint condition at the crack tip [i.e., the effect of interface normal (peel) and shear stresses on the deformations of sublayers is considered] of DCB specimen in conjunction with the first order shear deformation theory. But these SERR values from $\mathrm{HOBT}^{k}$ model, Bao et al. (1992) and Wang and Qiao (2005), are less accurate compared to the SERR values from Whitney (1985), Crews et al. (1991), and Olsson (1992) for small $a / h$ ratios. However, it can be seen that $\mathrm{HOBT} 2_{Q T}^{k}$ model (with nonvariationally derived matching condi- 
Table 3. Comparison of SERR Values from the Present Work (HOBT2) with Those from the Earlier Research Works for Various Crack Lengths of Unidirectional DCB Specimen

\begin{tabular}{|c|c|c|c|c|c|c|c|c|}
\hline \multirow[b]{2}{*}{ Model/theory } & \multicolumn{8}{|c|}{ Strain energy release rate $\times 10^{6}\left(\mathrm{~J} / \mathrm{m}^{2}\right)$} \\
\hline & \multicolumn{2}{|c|}{$\begin{array}{c}a=6.35 \mathrm{~mm} \\
(a / h=3.85)\end{array}$} & \multicolumn{2}{|c|}{$\begin{array}{c}a=12.7 \mathrm{~mm} \\
(a / h=7.7)\end{array}$} & \multicolumn{2}{|c|}{$\begin{array}{c}a=25.4 \mathrm{~mm} \\
(a / h=15.4)\end{array}$} & \multicolumn{2}{|c|}{$\begin{array}{c}a=50.8 \mathrm{~mm} \\
(a / h=30.8)\end{array}$} \\
\hline Crews et al. $(1991)^{a}$ & \multicolumn{2}{|c|}{1.77} & \multicolumn{2}{|c|}{4.96} & \multicolumn{2}{|c|}{16.2} & \multicolumn{2}{|c|}{57.2} \\
\hline Olsson- $G_{p \sigma}(1992)$ & \multicolumn{2}{|c|}{1.77} & \multicolumn{2}{|c|}{4.96} & \multicolumn{2}{|c|}{16.2} & \multicolumn{2}{|c|}{57.9} \\
\hline Olsson- $C_{f} G_{p \sigma}(1992)$ & \multicolumn{2}{|c|}{1.76} & \multicolumn{2}{|c|}{4.95} & \multicolumn{2}{|c|}{16.1} & \multicolumn{2}{|c|}{57.8} \\
\hline Whitney-Pl.o (1985) ${ }^{\mathrm{a}}$ & \multicolumn{2}{|c|}{1.72} & \multicolumn{2}{|c|}{4.88} & \multicolumn{2}{|c|}{16.0} & \multicolumn{2}{|c|}{57.6} \\
\hline Whitney-Pl.€ $(1985)^{\mathrm{a}}$ & \multicolumn{2}{|c|}{1.71} & \multicolumn{2}{|c|}{4.85} & \multicolumn{2}{|c|}{15.9} & \multicolumn{2}{|c|}{57.1} \\
\hline Wang and Qiao (2005) & \multicolumn{2}{|c|}{1.63} & \multicolumn{2}{|c|}{4.73} & \multicolumn{2}{|c|}{15.7} & \multicolumn{2}{|c|}{57.1} \\
\hline Bao et al. (1992) & \multicolumn{2}{|c|}{1.66} & \multicolumn{2}{|c|}{4.76} & \multicolumn{2}{|c|}{15.8} & \multicolumn{2}{|c|}{57.2} \\
\hline Present work & Pl. $\epsilon$ & Pl. $\sigma$ & Pl. $\epsilon$ & Pl. $\sigma$ & Pl. $\epsilon$ & Pl. $\sigma$ & Pl. $\sigma$ & Pl. $\sigma$ \\
\hline HOBT2 & 1.56 & 1.58 & 4.59 & 4.63 & 15.4 & 15.6 & 15.6 & 56.7 \\
\hline $\mathrm{HOBT}^{k}$ & 1.63 & 1.64 & 4.70 & 4.75 & 15.6 & 15.8 & 15.8 & 57.1 \\
\hline $\mathrm{HOBT} 2_{Q T}^{k}{ }^{\mathrm{b}}$ & 1.73 & 1.75 & 4.88 & 4.93 & 15.9 & 16.1 & 16.1 & 57.8 \\
\hline
\end{tabular}

${ }^{\mathrm{a}}$ SERR values as reported by Olsson (1992).

${ }^{\mathrm{b}}$ Nonvariationally derived matching conditions have been applied at the crack tip.

tions at the crack tip) gives comparable performance with Crews et al. (1991) and Olsson (1992) in determining the SERR for all the crack lengths including small $a / h$ ratios. This indicates that the application of nonvariationally derived matching conditions at the crack tip gives good performance than the application of variationally derived matching conditions at the crack tip, which is ironic. In essence, the HOBT2 model with variationally derived matching conditions at the crack tip $\left(\mathrm{HOBT}_{2}{ }^{k}\right.$ ) agrees with Wang and Qiao (2005) and the HOBT2 model with nonvariationally derived matching conditions at the crack tip $\left(\mathrm{HOBT}_{Q T}^{k}\right)$ agrees with Crews et al. (1991) and Olsson (1992) in determining the SERR for a unidirectional DCB specimen. Based on the above observations, it may be said that even though the present SERR solutions for multidirectional DCB specimen using HOBT2 models are very tedious; they can be useful as higher-order theory based analytical benchmarks for future simpler solutions.

\section{Parametric Study}

For the purpose of parametric study, symmetric multidirectional DCB specimen having $\left(0_{4}^{\circ} / 90_{4}^{\circ} / 0_{4}^{\circ} / \pm \theta_{2}\right)_{s}$ LS (32 laminae) made up of graphite/epoxy material has been considered. The material properties for graphite/epoxy are similar to those used in the multidirectional composite DCB specimen example of Table 2. The length, width, and thickness of the DCB specimen are $L=101.6 \mathrm{~mm}, b=25.4 \mathrm{~mm}$, and $2 h=4.064 \mathrm{~mm}$, respectively. The loading is $P=100 \mathrm{~N}$.

\section{Comparison between Plane Stress Analysis and Plane Strain Analysis}

Multidirectional $\left(0_{4}^{\circ} / 90_{4}^{\circ} / 0_{4}^{\circ} / \pm \theta_{2}\right)_{s}$ DCB specimen has been

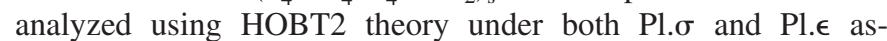
sumptions in the width direction to study the influence of fiber orientation on them. For a given crack length " $a / h=25$," Fig. 2 shows the compliance and SERR for various fiber orientations $\left(\theta=0^{\circ}, 15^{\circ}, 30^{\circ}, 45^{\circ}, 60^{\circ}, 75^{\circ}, 90^{\circ}\right)$. From Fig. 2, it can be observed that as the fiber orientation " $\theta$ " varies from $0^{\circ}$ to $90^{\circ}$, the compliance and SERR increase. Further, both in the case of compliance and SERR, Pl. $\sigma$ and Pl. $\epsilon$ analyses are close to each other only for the $0^{\circ}$ and $90^{\circ}$-fiber orientations and they differ significantly with each other for the fiber orientations other than $0^{\circ}$ and $90^{\circ}\left(\theta=15^{\circ}, 30^{\circ}, 45^{\circ}, 60^{\circ}, 75^{\circ}\right)$. To present further results, only the Pl.€ analysis has been considered because the performance of $\mathrm{Pl} . \epsilon$ analysis is good for both uni- and cross-ply laminates and moderate for multidirectional laminates.

\section{Influence of Crack Length (alh Ratio) on SERR}

Table 4 shows the influence of crack length $(a / h$ ratios $5-40)$ on the normalized SERR values for cross-ply $\left(0_{4}^{\circ} / 90_{4}^{\circ} / 0_{4}^{\circ} / 90_{4}^{\circ}\right)_{s}$ composite DCB specimen based on various shear deformation beam theories along with the SERR values from CBT. It may be noted that the SERR values from shear deformation models have been normalized with the SERR values from CBT model where ever they appear. It can be observed from Table 4 that as the crack length increases, SERR increases and the SERR values from $\mathrm{FOBT}^{k}, \mathrm{HOBT}^{k}, \mathrm{HOBT}_{2}{ }^{k}, \mathrm{HOBT} 1_{Q}^{k}$, and $\mathrm{HOBT} 2_{Q T}^{k}$ models ap-

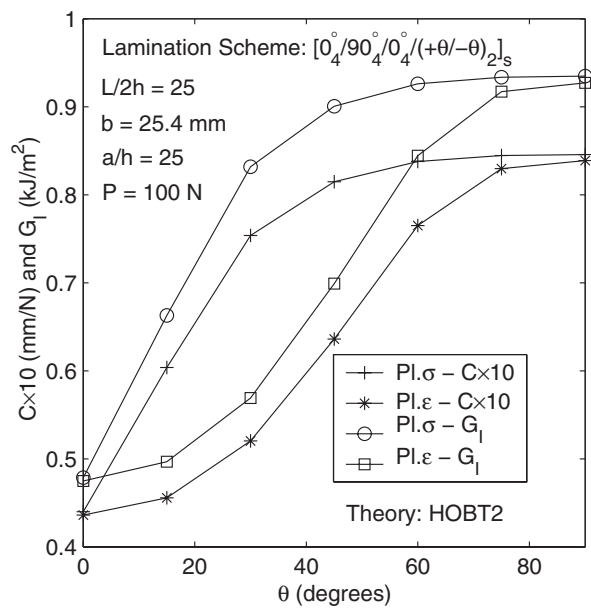

Fig. 2. Compliance and SERR values from Pl. $\sigma$ and Pl. $\epsilon$ analyses for various fiber orientations 
Table 4. Influence of Crack Length $(a / h)$ on SERR for Cross-Ply $\left(0_{4}^{\circ} / 90_{4}^{\circ} / 0_{4}^{\circ} / 90_{4}^{\circ}\right)_{s}$ Composite DCB Specimen Using Various Beam Theories-Plane Strain Analysis

\begin{tabular}{|c|c|c|c|c|c|c|}
\hline \multirow[b]{2}{*}{$a / h$} & \multirow{2}{*}{$\begin{array}{c}G_{I}^{\mathrm{CBT}} \\
\left(\mathrm{J} / \mathrm{m}^{2}\right) \\
\end{array}$} & \multicolumn{5}{|c|}{$G_{I} / G_{I}^{\mathrm{CBT}}$} \\
\hline & & $\mathrm{FOBT}^{k}$ & $\mathrm{HOBT}^{k}$ & HOBT $2^{k}$ & $\mathrm{HOBT}_{Q}{ }_{Q}{ }^{\mathrm{a}}$ & $\operatorname{HOBT}_{Q T}^{k}{ }^{\mathrm{a}}$ \\
\hline 5 & 33.5 & 1.5387 & 1.6087 & 1.6306 & 1.6931 & 1.7079 \\
\hline 10 & 134.2 & 1.2549 & 1.2863 & 1.2961 & 1.3238 & 1.3304 \\
\hline 15 & 301.9 & 1.1667 & 1.1869 & 1.1931 & 1.2109 & 1.2150 \\
\hline 20 & 536.6 & 1.1238 & 1.1387 & 1.1433 & 1.1563 & 1.1593 \\
\hline 25 & 838.5 & 1.0985 & 1.1102 & 1.1138 & 1.1241 & 1.1265 \\
\hline 30 & 1207.5 & 1.0818 & 1.0914 & 1.0944 & 1.1029 & 1.1049 \\
\hline 35 & 1643.5 & 1.0699 & 1.0781 & 1.0807 & 1.0879 & 1.0896 \\
\hline 40 & 2146.6 & 1.0610 & 1.0682 & 1.0704 & 1.0767 & 1.0782 \\
\hline
\end{tabular}

${ }^{\mathrm{a}}$ Nonvariationally derived matching conditions have been applied at the crack tip.

proach those from CBT model. Further, it can be said that the contribution of shear deformation is very significant for $a / h$ $<15$ and significant for $15 \leqslant a / h \leqslant 25$.

\section{Influence of $E_{11} / G_{13}$ Ratio on SERR}

Table 5 shows the influence of $E_{11} / G_{13}$ ratio (20-50) on the normalized SERR values for cross-ply $\left(0_{4}^{\circ} / 90_{4}^{\circ} / 0_{4}^{\circ} / 90_{4}^{\circ}\right)_{s}$ composite DCB specimen, having a crack length " $a / h=5$," based on various shear deformation beam theories along with the SERR values from CBT. From Table 5, it can be observed that SERR value from CBT is not influenced by $E_{11} / G_{13}$ ratio as CBT does not account for shear deformation. Next, as the $E_{11} / G_{13}$ ratio increases, SERR values/normalized SERR values from $\mathrm{FOBT}^{k}$, $\mathrm{HOBT}_{1}^{k}, \mathrm{HOBT}_{2}{ }^{k}, \mathrm{HOBT}_{Q}^{k}$, and $\mathrm{HOBT} 2_{Q T}^{k}$ models increase, which means that they deviate from CBT model's SERR values indicating that the significance of shear deformation increases as $E_{11} / G_{13}$ ratio increases.

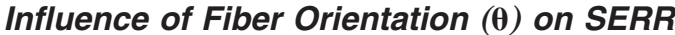

Table 6 shows the influence of fiber orientation $(\theta)$ on the normalized SERR values for multidirectional $\left(0_{4}^{\circ} / 90_{4}^{\circ} / 0_{4}^{\circ} / \pm \theta_{2}\right)_{s}$ composite DCB specimen, having a crack length " $a / h=5$," based on various shear deformation beam theories along with the SERR values from CBT. From Table 6 , it can be observed that as $\theta$ varies from $0^{\circ}$ to $90^{\circ}$, the SERR values increase and the normalized SERR values from $\mathrm{FOBT}^{k}, \mathrm{HOBT}^{k}, \mathrm{HOBT}^{k}, \mathrm{HOBT}_{2}^{k}$, and $\mathrm{HOBT}_{Q T}^{k}$ models decrease indicating the tendency of shear deformation models toward CBT as $\theta$ varies from $0^{\circ}$ to $90^{\circ}$.

\section{Interlaminar Normal Stress Distribution Ahead of the Crack Tip}

Fig. 3 shows the interlaminar normal stress distribution ahead of the crack tip using HOBT2 beam model for various fiber orientations of the DCB specimen having $\left(0_{4}^{\circ} / 90_{4}^{\circ} / 0_{4}^{\circ} / \pm \theta_{2}\right)_{s}$ LS and

Table 5. Influence of $E_{11} / G_{13}$ on SERR for $\left(0_{4}^{\circ} / 90_{4}^{\circ} / 0_{4}^{\circ} / 90_{4}^{\circ}\right)_{s}$ Cross-Ply Composite DCB Specimen $\left(E_{11}=146.86\right.$ GPa, $\left.a / h=5\right)$ Using Various Beam Theories-Plane Strain Analysis

\begin{tabular}{|c|c|c|c|c|c|c|}
\hline \multirow[b]{2}{*}{$E_{11} / G_{13}$} & \multirow{2}{*}{$\begin{array}{c}G_{I}^{\mathrm{CBT}} \\
\left(\mathrm{J} / \mathrm{m}^{2}\right)\end{array}$} & \multicolumn{5}{|c|}{$G_{I} / G_{I}^{\mathrm{CBT}}$} \\
\hline & & $\mathrm{FOBT}^{k}$ & $\mathrm{HOBT}^{k}{ }^{k}$ & $\mathrm{HOBT}^{k}$ & $\operatorname{HOBT}_{Q}^{k{ }^{a}}$ & $\operatorname{HOBT}_{Q T}^{k}{ }^{\mathrm{a}}$ \\
\hline 20 & 33.5 & 1.4871 & 1.5641 & 1.5889 & 1.6548 & 1.6651 \\
\hline 30 & 33.5 & 1.5571 & 1.6247 & 1.6457 & 1.7070 & 1.7235 \\
\hline 40 & 33.5 & 1.6051 & 1.6667 & 1.6856 & 1.7441 & 1.7655 \\
\hline 50 & 33.5 & 1.6402 & 1.6977 & 1.7154 & 1.7718 & 1.7974 \\
\hline
\end{tabular}

${ }^{\mathrm{a}}$ Nonvariationally derived matching conditions have been applied at the crack tip.

Table 6. Influence of Fiber Orientation $(\theta)$ on SERR for Multidirectional $\left(0_{4}^{\circ} / 90_{4}^{\circ} / 0_{4}^{\circ} / \pm \theta_{2}\right)_{s}$ Composite DCB Specimen $(a / h=5)$ Using Various Beam Theories-Plane Strain Analysis

\begin{tabular}{|c|c|c|c|c|c|c|}
\hline \multirow[b]{2}{*}{$\theta$} & \multirow{2}{*}{$\begin{array}{c}G_{I}^{\mathrm{CBT}} \\
\left(\mathrm{J} / \mathrm{m}^{2}\right)\end{array}$} & \multicolumn{5}{|c|}{$G_{I} / G_{I}^{\mathrm{CBT}}$} \\
\hline & & $\mathrm{FOBT}^{k}$ & HOBT $1^{k}$ & HOBT $2^{k}$ & $\operatorname{HOBT}_{Q}^{k}{ }^{a}$ & $\operatorname{HOBT} 2_{Q T}^{k}{ }^{\text {a }}$ \\
\hline $0^{\circ}$ & 16.6 & 1.7659 & 1.8434 & 1.8680 & 1.9398 & 1.9633 \\
\hline $15^{\circ}$ & 17.5 & 1.7474 & 1.8242 & 1.8486 & 1.9198 & 1.9426 \\
\hline $30^{\circ}$ & 20.1 & 1.6943 & 1.7692 & 1.7929 & 1.8623 & 1.8834 \\
\hline $45^{\circ}$ & 25.0 & 1.6213 & 1.6935 & 1.7162 & 1.7831 & 1.8017 \\
\hline $60^{\circ}$ & 30.4 & 1.5624 & 1.6325 & 1.6544 & 1.7190 & 1.7354 \\
\hline $75^{\circ}$ & 33.2 & 1.5406 & 1.6102 & 1.6320 & 1.6951 & 1.7103 \\
\hline $90^{\circ}$ & 33.5 & 1.5387 & 1.6087 & 1.6306 & 1.6931 & 1.7079 \\
\hline
\end{tabular}

${ }^{\mathrm{a}}$ Nonvariationally derived matching conditions have been applied at the crack tip. 


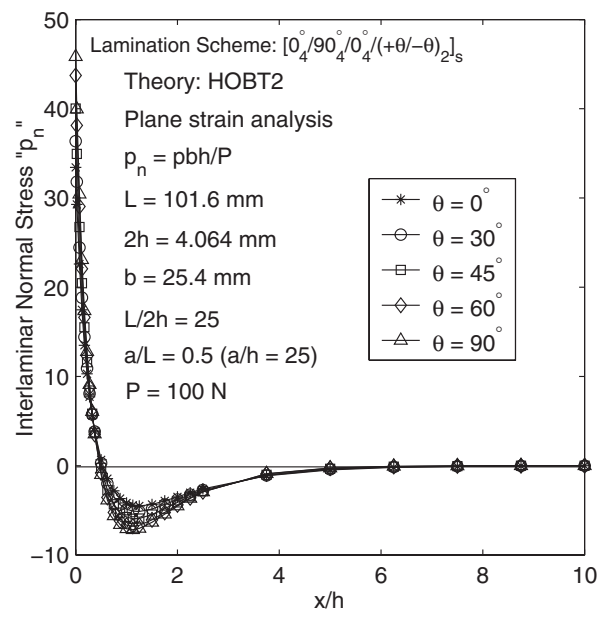

Fig. 3. Influence of fiber orientation on the interlaminar normal stress distribution ahead of the crack tip

crack length " $a / h=25$." From the Fig. 3 , it can be observed that as the fiber orientation " $\theta$ " varies from $0^{\circ}$ to $90^{\circ}$, the peak interlaminar normal stress at the crack tip increases and its decay length $(x / h)$ ahead of the crack tip to approach the 0 value is around 6 for all the fiber orientations.

\section{Concluding Remarks}

Mathematical modeling, for the analysis of symmetric multidirectional DCB specimen, has been presented using various laminated composite beam theories, namely, CBT, FOBT, SOBT, TOBT, HOBT1, and HOBT2. In the present study, both variationally and nonvariationally derived matching conditions have been applied at the crack tip of DCB specimen. For the unidirectional and cross-ply composite DCB specimens, the beam models under

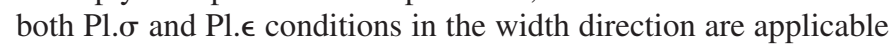
with good performance where as for the multidirectional composite DCB specimen, only the beam model under plane strain condition in the width direction appears to be applicable with moderate performance. Among the beam theories considered, the performance of higher-order shear deformation beam theory (HOBT2), having quadratic variation for transverse displacement over the thickness, is superior in determining the SERR solution for multidirectional DCB specimen. The present multidirectional DCB specimen stress analysis model "HOBT2" is applicable for the DCB specimens made up of unidirectional, symmetric crossply and symmetric multidirectional composite laminates, and gives good performance in determining the SERR and interlaminar normal stress distribution ahead of the crack tip for unidirectional and symmetric cross-ply composite DCB specimens. The HOBT2 model with variationally derived matching conditions at the crack tip (HOBT ${ }^{k}$ ) agrees with Wang and Qiao (2005) and the HOBT2 model with nonvariationally derived matching conditions at the crack tip $\left(\mathrm{HOBT}_{Q T}^{k}\right)$ agrees with Crews et al. (1991) and Olsson (1992) in determining the SERR for a unidirectional DCB specimen. Even though the SERR solutions for multidirectional DCB specimen using HOBT2 models are very tedious, they can be useful as higher-order theory based analytical benchmarks for future simpler solutions.

For the given LS, given crack length, and given load, the compliance, SERR, and peak interlaminar normal stress at the crack tip increase as the fiber orientation $\theta$ varies from $0^{\circ}$ to $90^{\circ}$. As the crack length increases, the SERR values from FOBT, SOBT, TOBT, HOBT1, and HOBT2 models approach those from CBT model. As the $E_{11} / G_{13}$ ratio increases, the SERR values from shear deformation beam models increase and also the significance of shear deformation increases. The effect of shear deformation on SERR is very significant for small $a / h$ ratios and large $E_{11} / G_{13}$ ratios. The decay length $(x / h)$ ahead of the crack tip, for the peak of the interlaminar normal stress to approach the 0 value, is around 6 for all the fiber orientations.

\section{Appendix. Stress-Strain Relations for Beam Theories}

\section{Stress-Strain Relations for НОВT1 and НОВТ2}

The stress-strain relations for HOBT1 and HOBT2 beam theories can be written, by reducing the transformed 3D stress-strain relations of an orthotropic lamina having arbitrary fiber orientation (Vinson and Sierakowski 1986) to suit to the beam models following the procedure explained by Pavan Kumar and Raghu Prasad (2008), as

$$
\sigma_{x x}=Q_{11} \epsilon_{x x}+Q_{13} \epsilon_{z z} ; \quad \sigma_{z z}=Q_{13} \epsilon_{x x}+Q_{33} \epsilon_{z z} ; \tau_{x z}=Q_{55} \gamma_{x z}
$$

in which $Q_{11}, Q_{13}, Q_{33}$, and $Q_{55}$ are defined as given below:

Plane stress condition

$$
\begin{gathered}
Q_{11}=\left\{\bar{C}_{11}+\bar{C}_{12} \frac{\left(\bar{C}_{16} \bar{C}_{26}-\bar{C}_{12} \bar{C}_{66}\right)}{\left(\bar{C}_{22} \bar{C}_{66}-\bar{C}_{26}^{2}\right)}+\bar{C}_{16} \frac{\left(\bar{C}_{12} \bar{C}_{26}-\bar{C}_{16} \bar{C}_{22}\right)}{\left(\bar{C}_{22} \bar{C}_{66}-\bar{C}_{26}^{2}\right)}\right\} \\
Q_{13}=\left\{\bar{C}_{13}+\bar{C}_{12} \frac{\left(\bar{C}_{26} \bar{C}_{36}-\bar{C}_{23} \bar{C}_{66}\right)}{\left(\bar{C}_{22} \bar{C}_{66}-\bar{C}_{26}^{2}\right)}+\bar{C}_{16} \frac{\left(\bar{C}_{23} \bar{C}_{26}-\bar{C}_{22} \bar{C}_{36}\right)}{\left(\bar{C}_{22} \bar{C}_{66}-\bar{C}_{26}^{2}\right)}\right\} \\
Q_{33}=\left\{\bar{C}_{33}+\bar{C}_{23} \frac{\left(\bar{C}_{26} \bar{C}_{36}-\bar{C}_{23} \bar{C}_{66}\right)}{\left(\bar{C}_{22} \bar{C}_{66}-\bar{C}_{26}^{2}\right)}+\bar{C}_{36} \frac{\left(\bar{C}_{23} \bar{C}_{26}-\bar{C}_{22} \bar{C}_{36}\right)}{\left(\bar{C}_{22} \bar{C}_{66}-\bar{C}_{26}^{2}\right)}\right\} \\
\text { and } Q_{55}=\left(\bar{C}_{55}-\frac{\bar{C}_{45}^{2}}{\bar{C}_{44}}\right)
\end{gathered}
$$

Plane strain condition

$$
Q_{11}=\bar{C}_{11}, Q_{33}=\bar{C}_{33}, Q_{13}=\bar{C}_{13}, \text { and } Q_{55}=\bar{C}_{55}
$$

where $\bar{C}_{i j}=$ transformed elastic stiffnesses.

\section{Stress-Strain Relations for CBT, FOBT, SOBT, and TOBT}

The stress-strain relations for CBT, FOBT, SOBT, and TOBT beam theories can be written, by reducing the transformed reduced two-dimensional stress-strain relations of an orthotropic lamina (including shear deformation) having arbitrary fiber orientation (Vinson and Sierakowski 1986) to suit to the beam models following the procedure explained by Pavan Kumar and Raghu Prasad (2008), as

$$
\sigma_{x x}=Q_{11}^{r} \epsilon_{x x} ; \tau_{x z}=Q_{55} \gamma_{x z}
$$

in which $Q_{11}^{r}$ and $Q_{55}$ are defined as given below: 
Plane stress condition

$$
\begin{aligned}
Q_{11}^{r}= & \left\{\bar{C}_{11}^{r}+\bar{C}_{12}^{r} \frac{\left[\bar{C}_{16}^{r} \bar{C}_{26}^{r}-\bar{C}_{12}^{r} \bar{C}_{66}^{r}\right]}{\left[\bar{C}_{22}^{r} \bar{C}_{66}^{r}-\left(\bar{C}_{26}^{r}\right)^{2}\right]}+\bar{C}_{16}^{r} \frac{\left[\bar{C}_{12}^{r} \bar{C}_{26}^{r}-\bar{C}_{16}^{r} \bar{C}_{22}^{r}\right]}{\left[\bar{C}_{66}^{r}-\left(\bar{C}_{26}^{r}\right)^{2}\right]}\right\} \\
& \text { and } Q_{55}=\left(\bar{C}_{55}-\frac{\bar{C}_{45}^{2}}{\bar{C}_{44}}\right)
\end{aligned}
$$

Plane strain condition

$$
Q_{11}^{r}=\bar{C}_{11}^{r} \text { and } Q_{55}=\bar{C}_{55}
$$

where $\bar{C}_{i j}^{r}=$ transformed reduced elastic stiffnesses.

\section{References}

Bao, G., Ho, S., Suo, Z., and Fan, B. (1992). "The role of material orthotropy in fracture specimens for composites." Int. J. Solids Struct., 29(9), 1105-1116.

Benbow, J. J., and Roesler, F. C. (1957). "Experiments on controlled fractures." Proc. Phys. Soc. London, Sect. B, 70, 201-211.

Broek, D. (1982). Elementary engineering fracture mechanics, Martinus Nijhoff, The Hague, The Netherlands.

Bruno, D., and Greco, F. (2001). "Mixed mode delamination in plates: A refined approach." Int. J. Solids Struct., 38(50-51), 9149-9177.

Chang, D. J., Muki, R., and Westmann, R. A. (1976). "Double cantilever beam models in adhesive mechanics." Int. J. Solids Struct., 12(1), 13-26.

Chatterjee, S. N., and Ramnath, V. (1988). "Modeling laminated composite structures as assemblage of sublaminates." Int. J. Solids Struct., 24(5), 439-458.

Crews, J. H., Jr., Shivakumar, K. N., and Raju, I. S. (1991). "Strain energy release rate distributions for double cantilever beam specimens." AIAA J., 29(10), 1686-1691.

Davidson, B. D., Krüger, R., and König, M. (1996). "Effect of stacking sequence on energy release rate distributions in multidirectional DCB and ENF specimens." Eng. Fract. Mech., 55(4), 557-569.

Davidson, B. D., and Schapery, R. A. (1988). "Effect of finite width on deflection and energy release rate of an orthotropic double cantilever specimen." J. Compos. Mater., 22(7), 640-656.

Davies, P., and Benzeggagh, M. L. (1989). "Interlaminar Mode I fracture testing." Composite Materials Series 6: Application of Fracture Mechanics to Composite Materials, Part IIA: Interlaminar Fracture Studies, Series, R. B. Pipes, ed., Elsevier Science, New York, 81-112.

Gilman, J. J. (1960). "Direct measurements of the surface energies of crystals." J. Appl. Phys., 31(12), 2208-2218.

Kanninen, M. F. (1973). "An augmented double cantilever beam model for studying crack propagation and arrest." Int. J. Fract., 9(1), 83-92.

Kanninen, M. F. (1974). "A dynamic analysis of unstable crack propagation and arrest in the DCB test specimen." Int. J. Fract., 10(3), 415430.

Khdeir, A. A., and Reddy, J. N. (1994). "Free vibration of cross-ply laminated beams with arbitrary boundary conditions." Int. J. Eng. Sci., 32(12), 1971-1980.

Li, S., Wang, J., and Thouless, M. D. (2004). "The effects of shear on delamination in layered materials." J. Mech. Phys. Solids, 52(1), 193 214.

Olsson, R. (1992). "A simplified improved beam analysis of the DCB specimen.” Compos. Sci. Technol., 43(4), 329-338.

Pavan Kumar, D. V. T. G. (1999). "Interlaminar fracture toughness of composites-Higher order shear deformation beam theories." Ph.D. thesis, Indian Institute of Science, Bangalore, India.

Pavan Kumar, D. V. T. G., and Raghu Prasad, B. K. (2003). "Higherorder beam theories for mode II fracture of unidirectional composites." ASME J. Appl. Mech., 70(6), 840-852.

Pavan Kumar, D. V. T. G., and Raghu Prasad, B. K. (2008). "Analysis of unidirectional DCB specimen using higher order beam theories." Eng. Fract. Mech., 75(8), 2156-2174.

Pradeilles-Duval, R.-M. (2004). "Quasi-static evolution of delaminated structures: analysis of stability and bifurcation." Int. J. Solids Struct., 41(1), 103-130.

Schön, J., Nyman, T., Blom, A., and Ansell, H. (2000). "A numerical and experimental investigation of delamination behaviour in the DCB specimen." Compos. Sci. Technol., 60(2), 173-184.

Sela, N., and Ishai, O. (1989). "Interlaminar fracture toughness and toughening of laminated composite materials: A review." Composites, 20(5), 423-435.

Sun, C. T., and Pandey, R. K. (1994). "Improved method for calculating strain energy release rate based on beam theory." AIAA J., 32(1), $184-189$.

Vinson, J. R., and Sierakowski, R. L. (1986). The behavior of structures composed of composite materials, Martinus Nijhoff, Dordrecht, The Netherlands.

Wang, J., and Qiao, P. (2004). "Interface crack between two shear deformable elastic layers." J. Mech. Phys. Solids, 52(4), 891-905.

Wang, J., and Qiao, P. (2005). "Analysis of beam-type fracture specimens with crack-tip deformation." Int. J. Fract., 132(3), 223-248.

Whitney, J. M. (1985). "Stress analysis of the double cantilever beam specimen." Compos. Sci. Technol., 23(3), 201-219.

Williams, J. G. (1988). "On the calculation of energy release rates for cracked laminates." Int. J. Fract., 36(2), 101-119.

Williams, J. G. (1989). "End corrections for orthotropic DCB specimens." Compos. Sci. Technol., 35(4), 367-376. 